\title{
ANÁLISE DA VIABILIDADE DE INVESTIMENTO EM MÁQUINAS E EQUIPAMENTOS PARA PRESTAÇÃO DE SERVIÇOS EM INDÚSTRIA DO SETOR SUCROENERGÉTICO
}

\author{
ANALYSIS OF INVESTMENT FEASIBILITY IN MACHINERY AND \\ EQUIPMENT FOR SERVICE IN SUGARCANE INDUSTRY.
}

Alessandro Antonio Tagliari Feba'; Alexandre da Silva Santana²; Willian Henrique Caetano da Silva³; Felipe Kesrouani Lemos*.

Universidade do Oeste Paulista - UNOESTE, Curso de Engenharia de Produção, Presidente Prudente, SP. e-mail: alefeba@hotmail.com, felipeklemos@unoeste.br.

RESUMO - Com a intensificação da mecanização agrícola no Brasil, ocorreu juntamente a este fato a evolução da produção da cana-deaçúcar. A região Centro-Sul abriga a maior demanda pela prestação dos serviços de tratos culturais. Assim, proporemos um estudo de viabilidade econômica e financeira de uma empresa do ramo de Cultivo de Cana-de-açúcar para ampliação da área a ser cultivada.

Palavras-chave: Prestação de serviços; Viabilidade; cultivo de cana-deaçúcar.

ABSTRACT - With the intensification of agricultural mechanization in Brazil, occurred along to this fact the development of production of cane sugar. The Center-South region has the highest demand for providing cultivation services. Thus, we propose a study of economic and financial viability of a company in the business of cultivation of cane sugar to expand the area to be cultivated.

Keywords: Services; viability; Sugar cane cultivation. 


\section{INTRODUÇÃO}

Tendo como base o estudo de Engenharia Econômica sob o ponto de vista de investimentos financeiros, a grosso modo, podemos inferir que investimento financeiro é uma aplicação de capital em meios de produção que visa o aumento da capacidade produtiva. Procura, a partir de então, auferir um retorno sobre os recursos investidos, de modo que seja, pelo menos, igual à taxa de juros ou que os lucros sejam maiores ou iguais ao capital investido.

Sobre a análise de investimentos, Rebelatto (2004, p. 141) explica que:

A Análise de Investimentos é um instrumento rápido, prático e seguro para auxiliar os profissionais de diversas áreas no processo de análise e tomada de decisões financeiras. Apresenta a Matemática Financeira como ciência básica e a Engenharia Econômica como técnica a ser aplicada.

A evolução da produtividade e da produção de cana-de-açúcar no Brasil, e em particular em nossa região, faz com que a demanda pela prestação dos serviços de tratos culturais cresça proporcionalmente. Assim, torna-se uma atividade promissora, mas que também necessita de melhores análises financeiras para a implementação de seus projetos de curto a longo prazo.

Nesse sentido, destacamos a essencial importância de uma análise econômico- financeira e uma boa utilização de suas ferramentas, aplicabilidade e resultados esperados.

\section{OBJETIVO}

O presente trabalho visa à realização de um estudo empírico sobre viabilidade econômica e financeira. Para tanto, escolhemos uma empresa do ramo de Cultivo de Cana-de-açúcar já constituída, e que hipoteticamente, tenha recebido uma proposta de trabalho de uma Indústria Sucroalcooleira, para ampliação da área a ser cultivada. A partir dessa proposta, serão levantados dados reais da empresa e projetados cenários realistas, pessimistas e otimistas. O contrato oferecido tem um prazo de 04 anos (safras), para cultivo de 13.000 ha $^{1}$ por safra. Para cumpri-lo será necessário investir em 03 tratores, 03 cultivadores, 01 caminhão Comboio, 01 caminhonete Oficina, 01 veículo para troca de turno e operadores e motoristas para tais veículos.

\section{JUSTIFICATIVA}

$O$ interesse por esse estudo surgiu em decorrência do trabalho da matéria de Engenharia Econômica como componente da avaliação bimestral, no qual foi verificado a viabilidade de investimento em máquinas e

\footnotetext{
${ }^{1}$ ha: simboliza hectare, que é uma unidade de medida de área equivalente a 100 (cem) ares ou a 10.000 (dez mil) metros quadrados.
} 
equipamentos à prestação de serviço em indústria do setor sucroenergético.

Os dados do trabalhos foram conseguidos pois um dos membros é coparticipante desta empresa que recebeu a proposta de ampliar sua amplitude de trabalhos.

Diante do exposto, o trabalho abordou métodos que possam auxiliar na tomada de decisão como o Método de Monte Carlos que se explica melhor no decorrer do trabalho.

\section{METODOLOGIA}

Será baseado, primeiramente, em uma breve revisão bibliográfica sobre engenharia econômica, sua importância e melhorias que pode trazer no processo decisório; sobre simulação, seu conceito e aplicações e a apresentação do cenário sobre a produção e produtividade da cana-deaçúcar no Brasil e na Região Centro-Sul, e por fim, apresentaremos as conclusões do projeto de investimento com a projeção do DRE e a análise de risco utilizando a simulação do Método de Monte $\mathrm{Carlo}^{2}$ (MMC).

\footnotetext{
${ }^{2}$ Método de Monte Carlo: de acordo com Taha (2008, p. 272), esta simulação compreende "um esquema de modelagem que estima parâmetros estocásticos ou determinísticos com base em amostragem aleatória".
}

\section{REVISÃO BIBLIOGRÁFICA}

\subsection{ENGENHARIA ECONÔMICA}

A Engenharia Econômica nos traz várias ferramentas de decisão e análise de investimentos. Possibilita realizarmos uma síntese econômica ou matemática às decisões de engenharia ou um corpo de conhecimentos e técnicas envolvidas na avaliação de bens de capital, bem como sua viabilidade econômico-financeira. Preocupase, basicamente, com o valor do dinheiro no tempo.

Para Ryba (2011, p.08), “Um dos principais aspectos da engenharia econômica é a decisão, ou escolha. Mais precisamente, onde e como fazer investimentos".

Nesse sentido, a Engenharia Econômica nos trará parâmetros suficientes para analisarmos o projeto aqui proposto.

\subsection{SIMULAÇÃO}

Simulação computacional juntamente com a otimização são ferramentas de fundamental importância no desenvolvimento de projetos, como a viabilidade de investimento em determinada aquisição e maquinários por exemplo.

Conforme Taha (2008, p.272), sobre simulação:

Simulação é o segundo melhor procedimento depois da observação de um sistema real. Trata de uma imitação computadorizada do comportamento 
aleatório de um sistema com a finalidade de estimar suas medidas de desempenho.

A simulação pode ser aproveitada nas mais variadas fases do desenvolvimento do projeto de viabilidade, entretanto é na fase de projeto que são tomadas as decisões mais importantes, respostas e perguntas como: Quantas máquinas comprar? Quantos turnos de trabalho serão necessários? São fundamentais para a formação adequada dos custos do novo projeto, sendo que a precisão desses números pode levar o novo projeto ao sucesso ou ao fracasso.
5.3 SETOR SUCROENERGÉTICO NO BRASIL E NA REGIÃO CENTRO-SUL

De acordo com dados do MAPA Ministério da Agricultura, Pecuária e Abastecimento, divulgados pela UDOP União dos Produtores de Bioenergia, o setor há uma crescente evolução da produtividade e da produção de cana-de-açúcar no Brasil. Observando o quadro abaixo, percebemos esse crescimento entre os períodos de safra $2005 / 2006$ a 2012/2013.

Tabela 1. Evolução da Produtividade e da Produção de Cana-de-Açúcar no Brasil por Ano-Safra.

\begin{tabular}{|c|c|c|c|c|c|c|c|c|c|}
\hline \multirow{2}{*}{$\begin{array}{c}\text { Ano-Safra } \\
\text { Harvest-Year }\end{array}$} & \multicolumn{3}{|c|}{ ÁREA (mil Hectares)" } & \multicolumn{3}{|c|}{ PRODUTIVIDADE (t/Ha) } & \multicolumn{3}{|c|}{ PRODUÇÃO (mil t)* } \\
\hline & \begin{tabular}{|c|} 
Centro-Sul \\
Center-South \\
\end{tabular} & $\begin{array}{l}\text { Norte / Nordeste } \\
\text { North / Northeast }\end{array}$ & $\begin{array}{l}\text { BRASIL } \\
\text { BRAZIL } \\
\end{array}$ & \begin{tabular}{c|} 
Centro-Sul \\
Center-South
\end{tabular} & $\begin{array}{l}\text { Norte / Nordeste } \\
\text { North / Northeast }\end{array}$ & $\begin{array}{l}\text { BRASIL } \\
\text { BRAZIL } \\
\end{array}$ & \begin{tabular}{c|} 
Centro-Sul \\
Center-South
\end{tabular} & \begin{tabular}{l|} 
Norte / Nordeste \\
North / Northeast \\
\end{tabular} & $\begin{array}{l}\text { BRASIL } \\
\text { BRAZIL } \\
\end{array}$ \\
\hline $2005 / 2006$ & $4.744,3$ & $1.096,0$ & $5.840,3$ & 70,4 & 44,1 & 65,5 & $334.136,6$ & $48.345,4$ & $382.482,0$ \\
\hline $2006 / 2007$ & $5.020,0$ & $1.143,3$ & $6.163,3$ & 74,5 & 48,0 & 69,6 & $373.912,9$ & $54.904,0$ & $428.816,9$ \\
\hline $2007 / 2008$ & $5.718,4$ & $1.227,9$ & $6.946,3$ & 75,4 & 52,6 & 71,4 & $431.233,5$ & $64.609,7$ & $495.843,2$ \\
\hline $2008 / 2009$ & $5.989,2$ & $1.068,7$ & $7.057,9$ & 84,9 & 60,0 & 81,1 & $508.638,8$ & $64.099,7$ & $572.738,5$ \\
\hline $2009 / 2010$ & $6.309,8$ & $1.099,8$ & $7.409,6$ & 86,0 & 54,8 & 81,4 & $542.825,0$ & $60.231,4$ & $603.056,4$ \\
\hline $2010 / 2011$ & $6.923,1$ & $1.132,5$ & $8.055,5$ & 81,0 & 56,0 & 77,5 & $561.037,4$ & $63.463,8$ & $624.501,2$ \\
\hline $2011 / 2012$ & $7.213,5$ & $1.148,9$ & $8.362,4$ & 68,6 & 57,5 & 67,1 & $494.937,7$ & $66.056,1$ & $560.993,8$ \\
\hline $2012 / 2013$ & $7.359,8$ & \begin{tabular}{|l|l|}
$1.125,2$ \\
\end{tabular} & $8.485,0$ & 72,5 & 49,5 & 69,4 & $533.517,6$ & $55.719,6$ & $589.237,1$ \\
\hline
\end{tabular}

Fonte: MAPA - Ministério da Agricultura, Pecuária e Abastecimento. Disponível em: http://www.udop.com.br/download/estatistica/area cultivada/14mai14 \%20area prodt brasil.pdf Acesso em 25/05/2014.

A partir da tabela, vemos que a área cultivada de cana-de-açúcar no Brasil passou de 5.840,3 (mil ha) na safra 2005/2006 para 8.485 (mil ha) na safra 2012/2013, ou seja, houve um aumento aproximado de 45,3\%. Já na região Centro-Sul, as áreas cultivadas passaram de 4.744,3 (mil ha) na safra 2005/2006 para 7.359,8 (mil ha) na safra
2012/2013, representando um aumento de $55,13 \%$.

A região Centro-Sul apresenta a maior área de cultivo de cana-de-açúcar do Brasil, com $86,7 \%$ do total, e o Estado de São Paulo representa $60 \%$ da área cultivada dessa região.

Analisando a figura abaixo, vemos uma diminuição da colheita manual 
queimada, e um aumento significativo da anos de 2003 e 2012.

colheita mecanizada de cana crua entre os

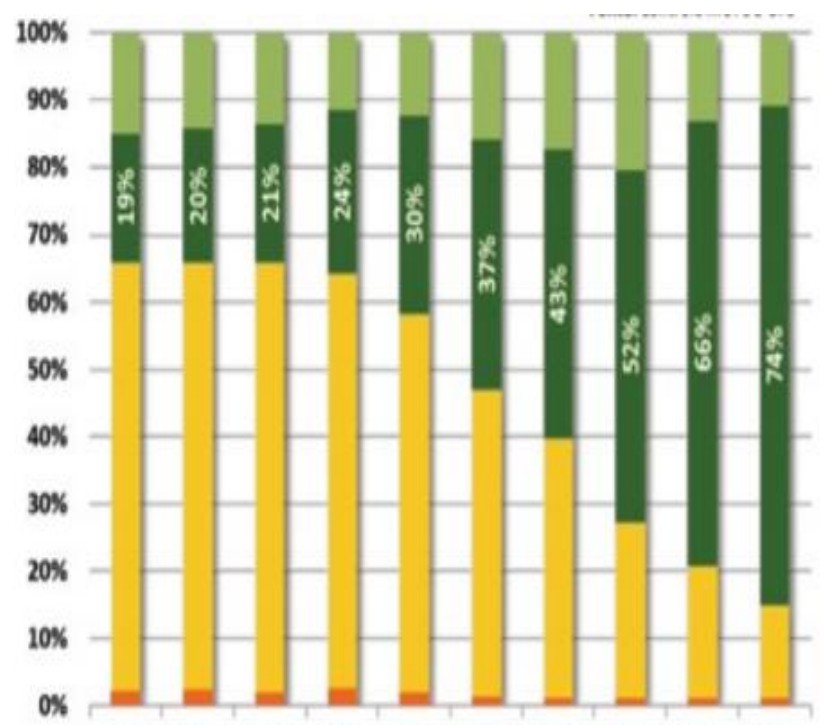

2003200420052006200720082009201020112012
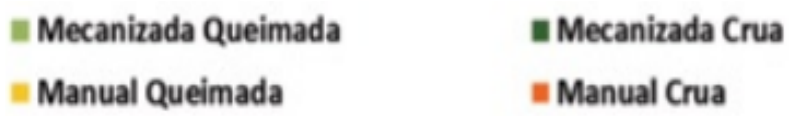

Figura 1. Distribuição percentual dos sistemas de colheita nas unidades da Região Centro-Sul, nas últimas dez safras.

Fonte:http://www.ctcanavieira.com.br/downloads/Censo2012.pdf

Atualmente, a colheita mecanizada da cana crua representa $74 \%$ do total cultivado no país. Isso se deve, principalmente, à Lei 11.241, de 19 de Setembro de 2002, que dispõe sobre a eliminação do processo de queima da palha de cana no período de précolheita. A partir dessa lei, os plantadores de cana devem buscar alternativas para um cultivo mais correto ambientalmente. E nesse contexto é que os empresários buscam investir em novas tecnologias e adquirir equipamentos mecanizados como colhedoras, tratores, transbordos, caminhões, cultivadores, etc.

A tabela abaixo apresenta essa evolução com os dados separados por tipo de colheita, onde podemos verificar 0 crescimento da colheita mecânica (cana crua e queimada) e diminuição da prática de colheita manual. 
Tabela 2. Evolução da mecanização agrícola na cana-de-açúcar na Região Centro-Sul, 2005_2012(em\%)

\begin{tabular}{lrrrrrrrrr}
\hline Centro-Sul & 2005 & 2006 & 2007 & 2008 & 2009 & 2010 & 2011 & 2012 \\
\hline Colleita mecânica - própria & 34,7 & 36,7 & 42,8 & 53,4 & 60,1 & 72,8 & 79,2 & 85,1 \\
Collheita crua - própria & 21,0 & 25,1 & 29,9 & 38,2 & 42,9 & 52,5 & 66,3 & 73,8 \\
$\begin{array}{l}\text { Collheita queimada - } \\
\text { própria }\end{array}$ & 13,7 & 11,6 & 12,9 & 15,2 & 17,1 & 20,3 & 13,0 & 10,8 \\
Colleita manulual - própria & 65,3 & 63,3 & 57,2 & 46,6 & 39,9 & 27,2 & 20,8 & 14,9 \\
Plantio mecânico-própria & & & 8,9 & 24,8 & 32,6 & 35,1 & 47,8 & 59,6 \\
\hline
\end{tabular}

Fonte: http://www.novacana.com/estudos/evolucao-plantio-colheita-mecanizados-cana-de-acucar-160813/

\section{MATERIAIS E MÉTODOS UTILIZADOS PARA}

\section{A CONFECÇÃO DO PROJETO}

Para o desenvolvimento do trabalho

foi feita uma breve revisão bibliográfica, abordando os conceitos de Engenharia Econômica, Simulação e à área de estudos, sendo esta o setor sucroenergético no Brasil e especialmente região Centro-Sul. Concomitantemente, foi elaborada uma planilha eletrônica contendo os dados da empresa estudada, em que traçaremos os cenários realista, otimista e pessimista, para no final calcularmos a $\operatorname{TIR}^{3}$ e o $\mathrm{VPL}^{4}$. Para análise de viabilidade econômico-financeira, utilizamos a ferramenta de programação do Excel denominada VBA - Visual Basic, com a Simulação de Monte Carlo.

\footnotetext{
${ }^{3}$ TIR: Taxa Interna de Retorno - conforme Samanez (2009, p.37), a TIR "objetiva encontrar uma taxa intrínseca de rendimento (...) é a taxa de retorno do investimento".

${ }^{4}$ VPL: Valor Presente Líquido - "mede o valor presente dos fluxos de caixa gerados pelo projeto ao longo de sua vida útil". (SAMANEZ. 2009, p. 36).
} 
Tabela 3. DRE - Demonstração do Resultado do Exercício projetada

\begin{tabular}{|c|c|c|c|c|c|c|}
\hline \multicolumn{7}{|c|}{ DRE projetada } \\
\hline & \multicolumn{2}{|c|}{ Realista } & \multicolumn{2}{|c|}{ Pessimista } & \multicolumn{2}{|c|}{ Otimista } \\
\hline Receita bruta & $\mathrm{R} \$$ & $1.079 .000,00$ & $\mathrm{R} \$$ & 899.166 .67 & $\mathrm{R} \$$ & $1.079 .000,00$ \\
\hline (-) Impostos sobre receita & $\mathrm{R} \$$ & $151.167,90$ & $\mathrm{R} \$$ & $125.973,25$ & $\mathrm{R} \$$ & $151.167,90$ \\
\hline Receita líquida & $\mathrm{R} \$$ & $927.832,10$ & $\mathrm{R} \$$ & $773.193,42$ & $\mathrm{R} \$$ & $927.832,10$ \\
\hline [-] Custos & $\mathbf{R}$ & 456.186 .25 & R* & 539.751 .00 & $\mathbf{R} \$$ & 303.783 .88 \\
\hline Combustivel tratores & $\mathrm{R} \$$ & $388.800,00$ & $\mathrm{R} \$$ & $447.120,00$ & $\mathrm{R} \$$ & $262.440,00$ \\
\hline Combustível Comboio & $\mathrm{R} \$$ & $5.040,00$ & $\mathrm{R} \$$ & $5.796,00$ & $\mathrm{R} \$$ & $3.402,00$ \\
\hline Combustivel caminhonete & $\mathrm{R} \$$ & $6.300,00$ & $\mathrm{R} \$$ & $9.832,50$ & $\mathrm{R} \$$ & $2.733,75$ \\
\hline Combustível veículo & $\mathrm{R} \$$ & $5.906,25$ & $\mathrm{R} \$$ & $8.032,50$ & $\mathrm{R} \$$ & $3.898,13$ \\
\hline Manutenção tratores d equipamentos & $\mathrm{R} \$$ & $36.540,00$ & $\mathrm{R} \$$ & $48.720,00$ & $\mathrm{R} \$$ & $24.360,00$ \\
\hline Manutenção Comboio & $\mathrm{R} \$$ & $2.100,00$ & $\mathrm{R} \$$ & $3.150,00$ & $\mathrm{R} \$$ & $1.050,00$ \\
\hline Manutenção caminhonete & $\mathrm{R} \$$ & $7.750,00$ & $\mathrm{R} \$$ & $10.850,00$ & $\mathrm{R} \$$ & $4.650,00$ \\
\hline Manutenção veículo & $\mathrm{R} \$$ & $3.750,00$ & $\mathrm{R} \$$ & $6.250,00$ & $\mathrm{R} \$$ & $1.250,00$ \\
\hline Mão de obra total & $\mathbf{R *}$ & 185.697 .79 & Rः & 182.881.12 & $\mathbf{R} \$$ & 124.670 .55 \\
\hline Mấo de obra tratores & $\mathrm{R} \$$ & $117.314,89$ & $\mathrm{R} \$$ & $114.498,22$ & $\mathrm{R} \$$ & $73.383,38$ \\
\hline Măo de obra Comboio & $\mathrm{R} \$$ & $26.393,40$ & $\mathrm{R} \$$ & $26.393,40$ & $\mathrm{R} \$$ & $19.795,05$ \\
\hline Mão de obra caminhonete & $\mathrm{R} \$$ & $26.393,40$ & $\mathrm{R} \$$ & $26.393,40$ & $\mathrm{R} \$$ & $19.795,05$ \\
\hline Mão de obra veículo & $\mathrm{R} \$$ & $15.596,10$ & $\mathrm{R} \$$ & $15.596,10$ & $\mathrm{R} \$$ & $11.697,08$ \\
\hline LA.JIDA & $\mathrm{R} \$$ & $285.948,06$ & $\mathrm{R} \$$ & $50.561,29$ & $\mathrm{R} \$$ & $499.377,67$ \\
\hline Depreciaçāo total & $\mathbf{R} \leqslant$ & 143.750 .00 & Rs & 143.750 .00 & $\mathbf{R}$ : & 143.750 .00 \\
\hline Depreciação tratores & $\mathrm{R} \$$ & $138.750,00$ & $\mathrm{R} \$$ & $138.750,00$ & $\mathrm{R} \$$ & $138.750,00$ \\
\hline Depreciação veículo & $\mathrm{R} \$$ & $5.000,00$ & $\mathrm{R} \$$ & $5.000,00$ & $\mathrm{R} \$$ & $5.000,00$ \\
\hline Lucro contábil & $\mathbf{R}$ : & 142.198 .06 & $-\mathbf{R} \leqslant$ & 93.188.71 & $\mathbf{R} \leqslant$ & 355.627 .67 \\
\hline
\end{tabular}

\begin{tabular}{|l|lr|lr|ll|}
\hline Fluso da operaģa & R\$ & $285.948,06$ & R\$ & $50.561,29$ & R\$ & 499.377,67 \\
\hline
\end{tabular}

\begin{tabular}{|c|c|c|c|c|c|c|}
\hline Ano & & $\mathrm{FCL}$ & & $\mathrm{Cl}$ & & $\mathrm{FCl}$ \\
\hline 0 & . $F$ & 7000000,00 & . $R$ & 7000000000 & . $R \$$ & 7000000000 \\
\hline 1 & R & $2855,948,06$ & R\$ & $50.561,29$ & R\$ & $499.377,67$ \\
\hline 2 & $R \$$ & $2855.948,06$ & Ris & $50.561,29$ & R\$ & $499,377,67$ \\
\hline 3 & $R \$$ & $2855.948,06$ & RA & $50.561,29$ & R\$ & $499.377,67$ \\
\hline 4 & R & $554.7180,06$ & R\$ & $262.561,29$ & R\$ & $839.997,67$ \\
\hline
\end{tabular}

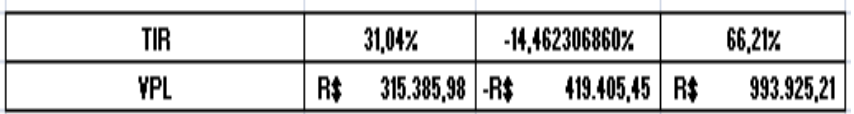

O método utilizado foi quantitativo, através do procedimento exploratório dos dados financeiros da empresa a ser analisada, bem como a projeção de custos e receitas a partir do investimento em equipamentos, máquinas agrícolas, caminhões, veículos e mão de obra especializada.
O método de coleta baseou-se em observações estruturadas, nas quais se procurou preservar os diferentes pontos de vista apresentados. O período de observação e coleta de informações foi realizado no início do mês de maio de 2014. 


\section{RESULTADOS E DISCUSSÕES}

A partir da análise dos dados tabulados no Excel, obtivemos os resultados dos cenários propostos, ou seja: realista, pessimista e otimista. Um dos fatores que mais contribui para o resultado dos cenários é a velocidade de cultivo, sendo que, para atender aos 13.000 ha propostos em 09 meses, é necessário que a velocidade seja de, no mínimo, 3 ha por hora, se for menor não atingirá e impactará diretamente na receita final, e consequentemente na TIR e VPL. Por outro lado, se a área cultivada for maior do que 3 ha, seremos mais eficientes, terminaremos antes do prazo e os custos serão reduzidos.

As variáveis aleatórias da nossa análise são: valor residual, velocidade de cultivo, percentual de tempo cultivando, manutenção e preço dos combustíveis. Desse modo, quando inserimos os valores reais e traçamos o cenário realista, a TIR resultou em $31,04 \%$ e o VPL em $R \$ 315.385,98$. No cenário otimista, mas que também é possível atendido durante os 09 meses de safra, a TIR ficou em 66,21\% e o VPL em R\$ 993.925,21. Já, no entanto, no cenário pessimista, considerando um combustível preço mais alto, manutenções constantes, tempo de cultivo menor e menor área cultivada por dia, a TIR ficou em $-14,46 \%$ e o VPL em -R\$
419.405,45, dando resultado negativo para o investimento.

Além de traçar os cenários otimista, pessimista e realista, foi feita uma simulação de Monte Carlo para avaliar o efeito da aleatoriedade combinada das variáveis probabilísticas. A Tabela 4 mostra os resultados obtidos com 1.000 simulações, expressos em termos da TIR.

Tabela 4. Resultados da simulação

\begin{tabular}{cc}
\hline Média & $22,91 \%$ \\
\hline Desvio & $16,96 \%$ \\
\hline$\%$ de insucessos & $32,80 \%$ \\
\hline Mínimo & $-20,31 \%$ \\
\hline Máximo & $52,72 \%$ \\
\hline
\end{tabular}

Com a análise e simulação de Monte Carlo, podemos inferir probabilidades estatísticas para determinar, a partir de 2 a milhares de simulações, se a TIR será ou não positiva. Em nossas verificações, a maior parte dos resultados obtidos ficou na faixa de $10 \%$ a $20 \%$, mostrando como uma viabilidade economicamente positiva. A Figura 2 mostra a distribuição obtida nas simulações para cada faixa da TIR. 


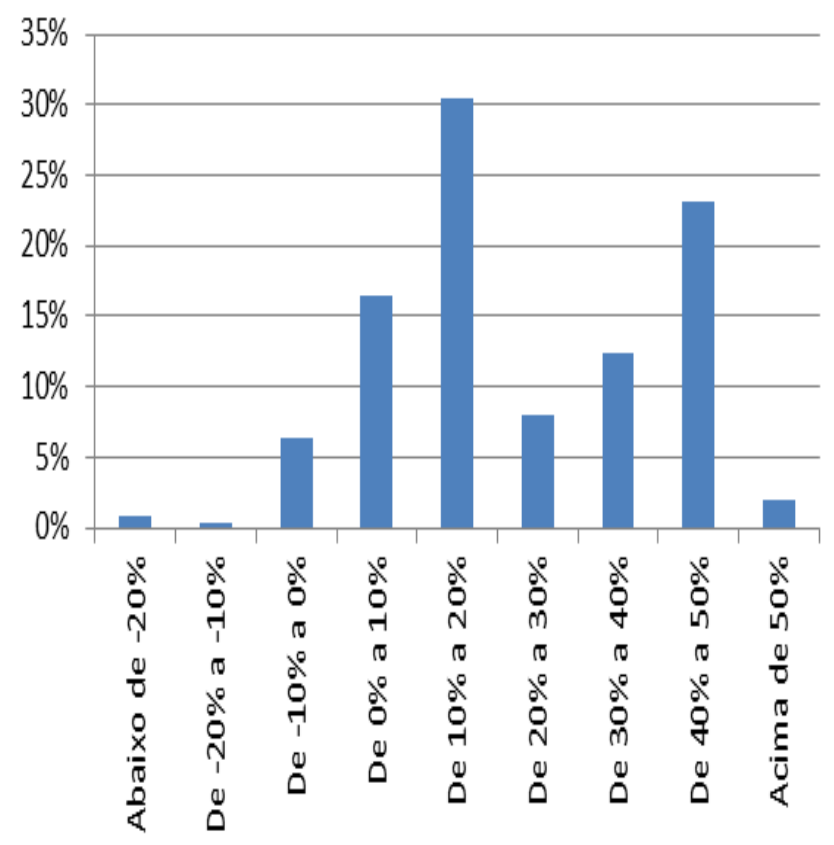

Figura 2. Resultado da frequência obtida em cada faixa da TIR

Os dados obtidos na simulação mostram que a simulação oferece um melhor posicionamento do risco do investimento. Enquanto as análises pessimista e otimista na modelagem inicial são extremas, na simulação é avaliado o efeito da aleatoriedade combinada das variáveis. O percentual de insucesso obtido foi de $32,8 \%$, havendo uma média de 16,96\%, ou seja, ligeiramente pior que o cenário realista idealizado.

\section{CONSIDERAÇÕES FINAIS}

Com base nos dados coletados e retrabalhados no projeto, concluímos que 0 projeto apresenta-se viável economicamente. Assim, dois fatores foram predominantes para tal conclusão, o VPL e a TIR, que em todas as simulações (pelo método de Monte Carlo) resultaram positivamente, em comparação com a taxa de atratividade aplicada no mercado atual.

Quanto à análise pelo $\mathrm{MMC}$, verificouse que as variáveis aleatórias escolhidas com intervalos pré-definidos se comportaram de maneira tal que corroboraram a ideia do modelo inicial de que o investimento continuasse sendo atrativo. Comparando os índices mostrados pela Simulação de Monte Carlo com o rendimento da poupança, mesmo assim o projeto torna-se atrativo.

Com base nos dados das simulações, verificou-se que o projeto torna-se viável em cerca de $2 / 3$ das simulações. Se considerarmos que existem investidores mais arrojados e outros mais conservadores, as possibilidades de sucesso são bastante encorajadoras. 
Outro fator relevante que deve ser analisado pelos investidores é o horizonte do planejamento, neste caso, quatro anos. Durante esse prazo, com certeza, ocorrerão imprevistos que devem ser ponderados nas análises do planejamento, é de suma importância o acompanhamento do planejamento, uma vez que o projeto seja implementado.

Por outro lado, existe sempre um risco neste tipo de negócio, quer seja climático, quer seja do comportamento do mercado financeiro, enfim, como em todo empreendimento, tem-se que assumir riscos calculados como foi realizado nesse projeto.

A partir dos cenários traçados, o projeto só não irá consumar-se como positivo, se todas as nossas variáveis aleatórias contribuírem negativamente, $\mathrm{Ou}$ seja, é muito mais provável que a partir do cenário realista cheguemos ao otimista, do que cairmos para o pessimista.

Portanto, concluímos que o projeto econômico-financeiro da empresa de cultivo de cana-de-açúcar caminhou-se para índices de TIR e VPL positivos, sendo assim, um projeto viável.

\section{AGRADECIMENTOS}

A realização do presente trabalho apenas foi possível devido a colaboração dos membros e em especial do orientador Professor Mestre Felipe Kesrouani Lemos, pelo prazer, disponibilidade e orientação que mais uma vez nos proporcionou nesse nosso crescimento científico e que não mediu esforço ao compartilhar seus conhecimentos, contribuindo imensamente na elaboração do presente trabalho, o nosso muito obrigado.

\section{REFERÊNCIAS}

BRASIL. Ministério da Agricultura, Pecuária e Abastecimento. Secretaria de Produção e Agroenergia. Disponível em: http://www.udop.com.br/download/estatisti ca/area cultivada/14mai14 \%20area prodt brasil.pdf. Acesso em 25/05/2014.

CTC - Centro de Tecnologia Canavieira. Censo Varietal e de Produtividade em 2012: Região Centro-Sul. Disponível em: http://www.ctcanavieira.com.br/downloads/ Censo2012.pdf. Acesso em 25/05/2014.

GOLDBARG, Marco Cesar; LUNA, Henrique Pacca. Otimização Combinatória e Programação Linear. Rio de Janeiro: Editora CAMPUS, 2000.

REBELATTO, Daisy (Coord.). Projeto de Investimento. Barueri, SP: Manole, 2004.

RYBA, Andréa; LENZI, Ervin Kamoinski; LENZI, Marcelo Kaminstki. Elementos de engenharia econômica. Curitiba: Ibpex, 2011.

SAMANEZ, Carlos Patrício. Engenharia Econômica. São Paulo: Pearson Prentice Hall, 2009.

TAHA, Hamdy A. Pesquisa Operacional: uma visão geral. 8. ed. São Paulo: Pearson Prentice Hall, 2008. 